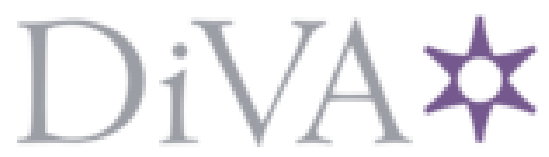

http://www.diva-portal.org

This is the published version of a paper presented at IEEE PQ 2014.

Citation for the original published paper:

Almas, M., Kilter, J., Vanfretti, L. (2014)

Experiences with Steady-State PMU Compliance Testing using Standard Relay Testing Equipment.

In:

N.B. When citing this work, cite the original published paper.

Permanent link to this version:

http://urn.kb.se/resolve?urn=urn:nbn:se:kth:diva- 157125 


\title{
Experiences with Steady-State PMU Compliance Testing using Standard Relay Testing Equipment
}

\author{
M. S. Almas, Student Member, IEEE, Jako Kilter, Member, IEEE, and Luigi Vanfretti, Member, IEEE
}

\begin{abstract}
This paper presents the results of steady state compliance testing of phasor measurement units (PMUs) from three different vendors in a laboratory environment. Testing is performed by providing three phase voltage and current injections to the VT and CT inputs of the PMUs through Freja-300 standalone protection relay test set. Testing is performed according to the standard "IEEE C37.242-2013 - IEEE Guide for Synchronization, Calibration, Testing, and Installation of Phasor Measurement Units (PMUs) for Power System Protection and Control". The paper discusses the test setup, testing process and overall test results of this project. The limitations of stand-alone testing equipment and recommendations for utilizing real-time hardware-in-the-loop simulation for thorough testing of PMUs are also presented.
\end{abstract}

Index Terms-Phasor Measurement Units, PMU calibration testing, stand-alone testing, synchrophasors.

\section{INTRODUCTION}

Phasor Measurement Units (PMUs) are increasingly being deployed around the world. PMUs are considered as one of the key technologies for wide area power system monitoring, protection and control (WAMPAC) systems [1]. Some of the potential applications of synchrophasors include state estimation, event and fault detection, oscillation monitoring, situation awareness and model validation [2]. It is foreseen that the use of PMUs and development of sophisticated control algorithms for system control purposes in future power systems will increase system security, and thus, an understanding of technical performance of PMUs is essential.

The reliability of power system applications based on synchrophasors leans on the accuracy of PMUs for phasor calculation and frequency estimation. The IEEE standard for Synchrophasor Measurements for Power Systems (IEEE C37.118.1-2011) [3] specifies requirements for PMUs for both steady state and dynamic operating conditions. The companion standard IEEE C37.118.2-2011 covers communication aspects of synchrophasor data transfer. Another standard "IEEE C37.242-2013 - IEEE Guide for Synchronization, Calibration, Testing, and Installation of Phasor Measurement Units (PMUs) for Power System Protection and Control" [4] provides guidance for test and calibration procedures for PMUs

This work was supported in part by Nordic Energy Research through the STRONg ${ }^{2}$ rid project and by Statnett SF, the Norwegian TSO. Support from Elering AS, Estonian TSO, is appreciated.

J. Kilter is with Tallinn University of Technology, Tallinn, Estonia and Estonian TSO Elering. (email: jako.kilter@ttu.ee, jako.kilter@elering.ee)

M. S. Almas, and L. Vanfretti are with KTH Royal Institute of Technology, Stockholm, Sweden. (e-mail: \{msalmas, luigiv\}@kth.se)

L. Vanfretti is with Statnett SF, Research and Development, Oslo, Norway (email: luigi.vanfretti@statnett.no) for laboratory and field applications. This paper presents results from static compliance testing of PMUs from three different vendors as specified by the above mentioned standards.

The remainder of the paper is organized as follows: Section II provides information about the testing equipment and the architecture for PMU testing. Section III presents results for steady state compliance tests for PMUs from three different vendors. Section IV identifies the limitations of standalone testing equipment and provides recommendations for a more thorough test bed for PMUs testing. Finally in Section $\mathrm{V}$, conclusions are drawn and experiences together with lesson learnt from the project are summarized.

\section{PMU Testing ARChitecture}

This section presents the details of the testing equipment, Freja-300 [5], used for this study. Freja-300 is a standard stand-alone test set intended for protection relay testing. This test set can generate $4 \times 150 \mathrm{~V}(82 \mathrm{VA})$ and $3 \times 15 \mathrm{~A}(87 \mathrm{VA})$ or $1 \times 45 \mathrm{~A}(250 \mathrm{VA})$. The inaccuracy guaranteed for the voltage and current generations by the test set is $( \pm 0.01 \%$ of range $)+( \pm 0.05 \%$ of reading $)$ which is within the accuracy limits for testing equipment specified by IEEE C37.242-2013 [4] which states that the uncertainty of test equipment should be lesser than $10 \%$ of the allowed error.

Freja-300 facilitates injecting harmonics, faults and unbalanced voltage / currents / frequency to the device under test. For most of the calibration testing scenarios, the test set is used in a sequence mode which allows making the settings for magnitude and phase angles along with frequency of current/voltage injections independently for up to 28 sequences which follow each other depending upon the time specified for each sequence. Sequence 1 is considered as a pre-fault or steady state sequence and Sequence 2 is the fault scenario where a disturbance is injected to the device under test to perform its dynamic testing.

The steady state tests performed includes Total Vector Error (TVE), magnitude error, and phase error at nominal frequency input at a PMU reporting rate of 50 frames per second. TCP is used as transport layer protocol for streaming out synchrophasors from PMUs over Ethernet in this study. In order to perform dynamic testing of the PMU under test, at least two sequences are required: a steady state sequence for a specific period of time followed by the disturbance sequence which can be step change in voltage/current magnitude or phase angles along with frequency change. Results for dynamic compliance tests will be presented in a separate publication.

The three phase voltage and current injections are provided to the voltage and current input modules of the PMU under test. The voltage and current injections are set by using soft- 
ware interface of Freja-300 on the workstation which is serially connected to the test set. The PMU receives the analog inputs and streams out synchrophasor measurements through its Ethernet port. For all the PMUs, synchrophasors for positive sequence and all phases for both voltage and currents were acquired. The PMU measurements are received in a Phasor Data Concentrator (PDC) to archive all the synchrophasor measurements as a CSV file for further analysis using MATLAB. The overall test setup is shown in Fig. 1.

The injection parameters are set by using Freja Win [6] on the workstation which is serially connected to the test set. The PMU under test receives the three phase voltage and current analog signals at its VT and CT input modules. The PMU is time synchronized by acquiring IRIG-B [7] signals from sub- station clock [8] which is connected to the GPS antenna. PMU computes the synchrophasors and streams them out through its Ethernet port. For all the PMUs, synchrophasors for positive sequence and all phases for both voltage and currents are acquired. The PMU measurements are received in a Phasor Data Concentrator SEL-5073 from Schweitzer Engineering Laboratories. The purpose of the PDC is to archive all the synchrophasor measurements as a CSV file for further analysis. Some commercial protection relay test sets do have the provision to send time synchronization signals to the device under test in order to synchronize the whole testing process. However this feature is not available in Freja-300 test set and therefore IRIG-B signals were provided to the PMU by using substation clock.

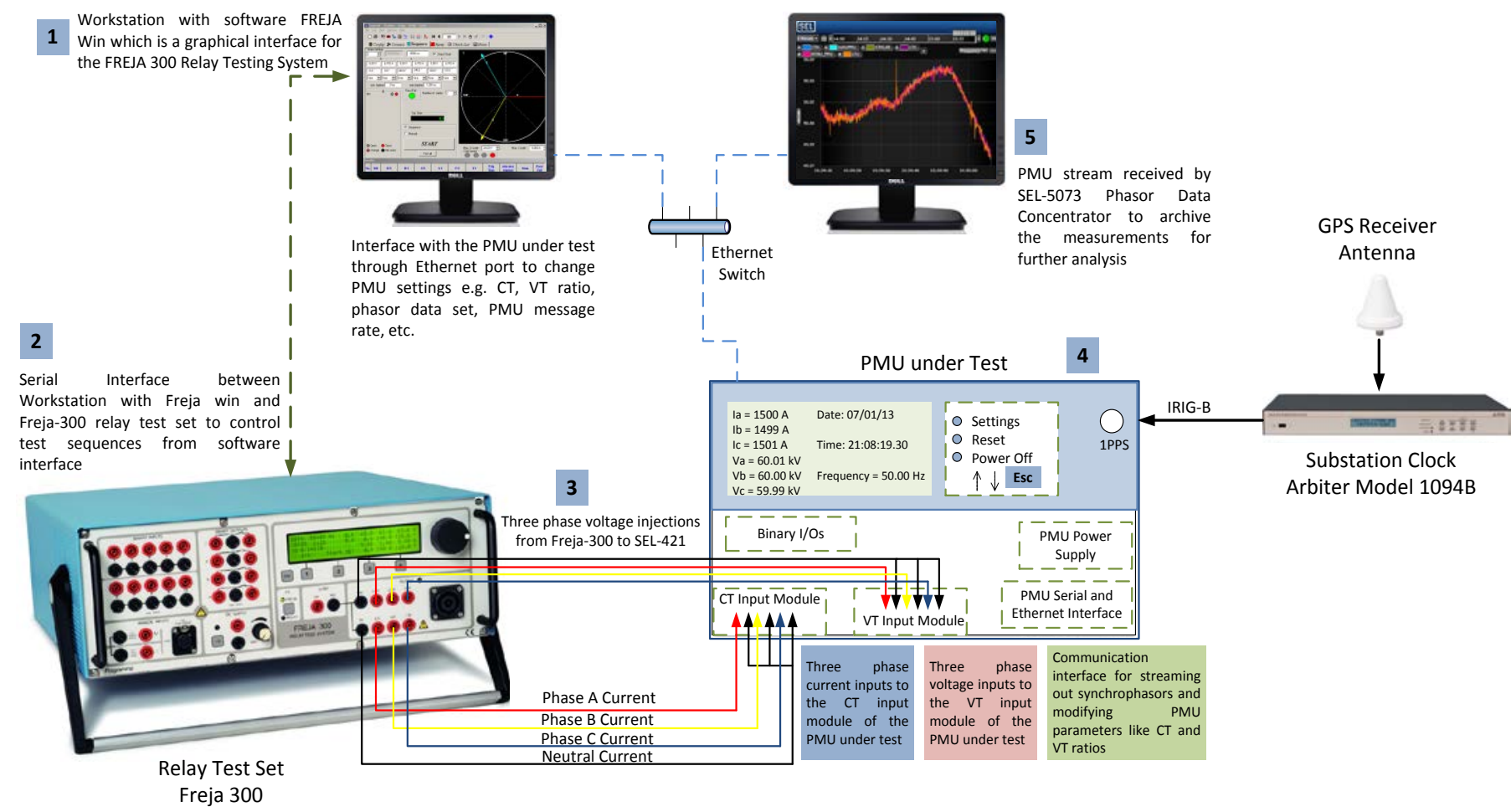

Fig. 1. PMU calibration test environment showing injection of three phase voltages and current to the PMU under test using Freja-300 Protection Relay Test Set. PMU measurements from the device under test are received in SEL-5073 Phasor Data Concentrator to archive the measurements as CSV files for future analysis.

\section{PMU TESTing Results}

This section presents the results obtained from steady state compliance testing of PMUs from three different vendors. The standard IEEE C37.118.1 [3] defines the steady state tests as the one in which the test signal does not vary with time. The steady state conformance tests performed in this study involve frequency variation, magnitude variation, phase variation, off nominal frequency response and harmonic distortion tests. The out of band interference test has not been performed in this study. An important factor for evaluating steady state conformance of PMUs is the Total Vector Error (TVE) which defines the uncertainty requirements for the PMUs and is calculated as

$$
\operatorname{TVE}(n)=\sqrt{\frac{\left(\hat{X}_{r}(n)-X_{r}(n)\right)^{2}+\left(\hat{X}_{i}(n)-X_{i}(n)\right)^{2}}{\left(X_{r}(n)\right)^{2}+\left(X_{i}(n)\right)^{2}}}
$$

where $\widehat{X}_{r}(n)$ and $\widehat{X}_{i}(n)$ are sequences of estimates given by the unit under test, and $X_{r}(n)$ and $X_{i}(n)$ are sequences of input signals from the test set Freja-300.

The TVE factor assures that the PMUs uncertainty in both magnitude and time synchronization error is bounded within a certain limit. This limit is specified in the standard to $1 \%$ and corresponds to a phase angle error of $0.573^{0}$ (degrees) or a time synchronization inaccuracy of $31.8 \mu$ s at $50 \mathrm{~Hz}$. 
Similarly, the standard defines frequency measurement error (FE) and rate of change of frequency measurement error (RFE) as

$$
\begin{aligned}
& F E=\left|f_{\text {true }}-f_{\text {measured }}\right|=\left|\Delta f_{\text {true }}-\Delta f_{\text {measured }}\right| \\
& R F E=\left|(d f / d t)_{\text {true }}-(d f / d t)_{\text {measured }}\right|
\end{aligned}
$$

where $f_{\text {measured }}$ is the frequency measured by PMU while $f_{\text {true }}$ is the frequency of the test signal generated by Freja-300

The standard IEEE C37.118.1 [3] allows the maximum permissible tolerance of $\pm 0.005 \mathrm{~Hz}$ in frequency error $(\mathrm{FE})$ and $\pm 0.01 \mathrm{~Hz} / \mathrm{sec}$ for rate of change of frequency (ROCOF) measurement error (RFE).

In this study, the performance of PMUs under steady state is evaluated for both a balanced three phase system and for a system with harmonic contents. The PMUs are graded pass or fail with reference to their fulfilment of the criteria for TVE, FE and RFE for both the test cases. All the tests are performed at test signal frequency of $50 \mathrm{~Hz}$ and the PMU reporting rate of 50 frames per second. The steady state tests at off nominal frequencies will be presented as separate publication.

\section{A. Steady-State Balanced Three Phase Test}

Fig. 2 shows the plots for TVE computed for both positive sequence and phase measurements of voltage (plots a-c) and current magnitudes (plots d-f) for the three PMUs. The results show that all the PMUs fulfil the requirement of TVE i.e. less than $1 \%$ in magnitude for steady state balanced three phase systems. However TVE for all the three phases are not same.

Fig. 3 shows the results for both frequency measurement error (FE) and rate of change of frequency (ROCOF) measurement error (RFE) as defined in (2). All the PMUs satisfy the steady state frequency error criteria (within $\pm 0.005 \mathrm{~Hz}$ ).
However one of the PMU violates the limit for RFE based on ROCOF calculations. The IEEE C37.118 standard also sets requirements for voltage and current phase angles accuracy which corresponds to $0.573^{\circ}$. The measurement for the phase angles is synchronized by the Pulse Per Second (PPS) provided by GPS or modulated signals like IRIG-B from substation clock. The inaccuracy in time synchronization is reflected as an error in phase angle computation by the PMU. GPS receiver malfunctioning, wrong orientation of receiver's antenna, time delay incorporated due to lengthy cable between GPS antenna and PMU, electromagnetic interference due to a neighbouring installations, GPS jamming, etc.; can result in time synchronization inaccuracy and thus lead to errors in the phase angle computations performed by the PMU. The substation clock used to provide time synchronization signals to the PMUs in these experiments has an average accuracy of $100 \mathrm{~ns}$ [8] which corresponds to a phase angle uncertainty of $0.0018^{\circ}$ (degrees) which is well below the 1\% TVE requirement.

Fig. 4 shows the phase angle TVE for positive sequence voltage and current as computed by all the PMUs when subject to steady state balanced three phase voltage and current injections. Phase angle TVE for both current and voltage are well within the permissible limit of $0.573^{\circ}$. The voltage phase angle TVE are much lesser than the current phase angle TVE for all the PMUs. These TVE calculations include the time delay incurred by the cables coupling Freja-300 test set with the PMU. Once this time delay induced by power cables is deterministically calculated, this delay can be compensated inside the PMUs' voltage and current phase angle calculations by accessing PMUs' settings. On the basis of these results, under steady state balanced three phase conditions, PMU A and PMU B fulfil all the requirements of TVE, FE and RFE while PMU C violates the requirements for RFE.
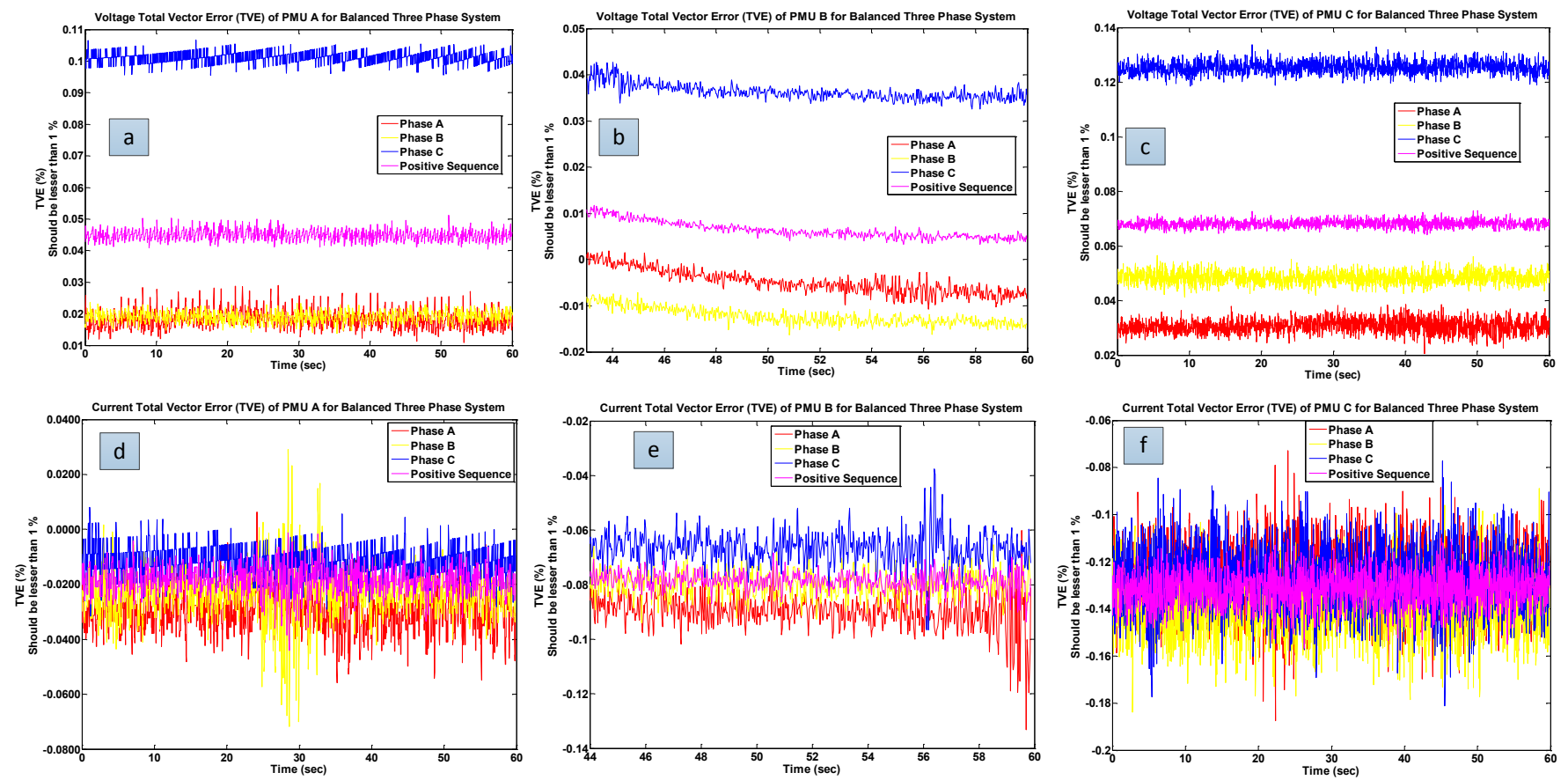

Fig. 2. Voltage and current TVE computed from the measurements of three PMUs under test in steady state conditions using (1). Three phase balanced voltages and currents of $60 \mathrm{~V}$ and 1 Ampere respectively with phase rotation abc is fed to the VT and CT inputs of the PMUs having VT ratio of 1000:1 and CT ratio of 1500:1. Measurements show that all the PMUs are well within the TVE limit of $1 \%$ for both voltage (plots a-c) and currents (plots d-f). 

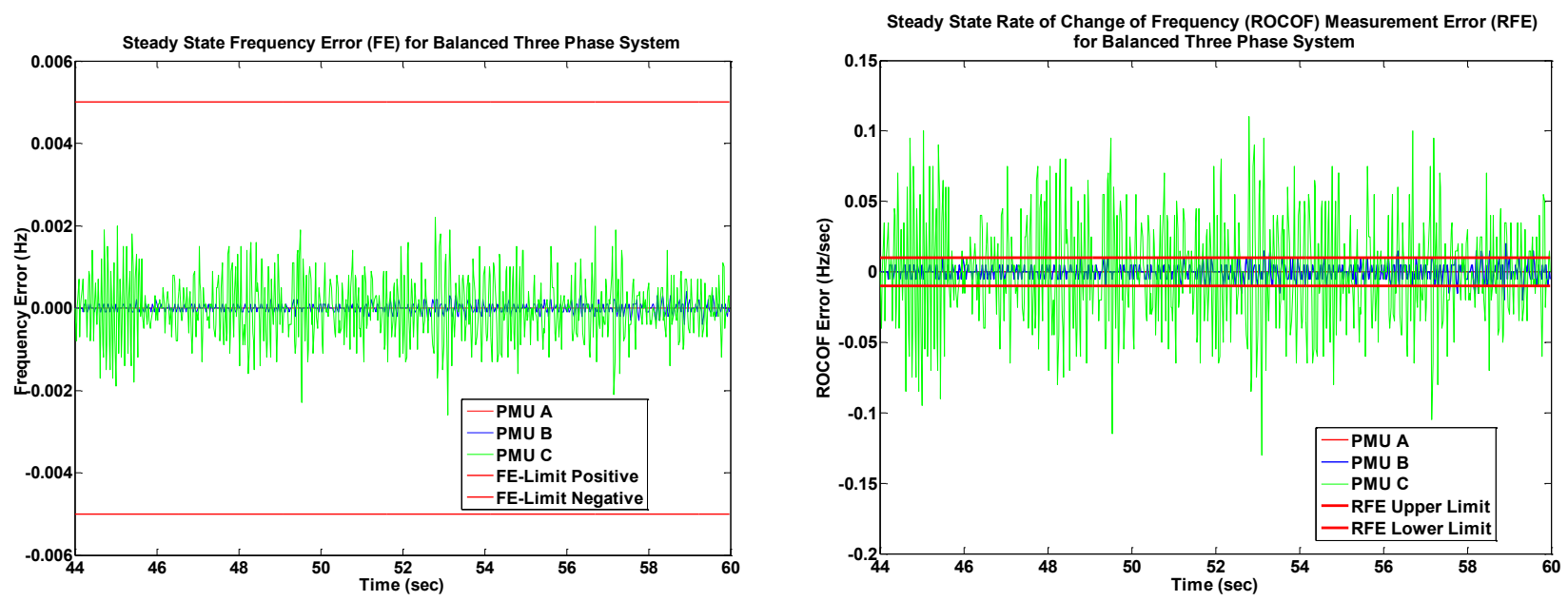

Fig. 3. Frequency Error (FE) and Rate of Change of Frequency (ROCOF) measurement error (RFE) calculated by using (2). All the PMUs satisfy the requirement for frequency error within $\pm 0.005 \mathrm{~Hz}$. Only one PMU does not fulfil the requirement for RFE.
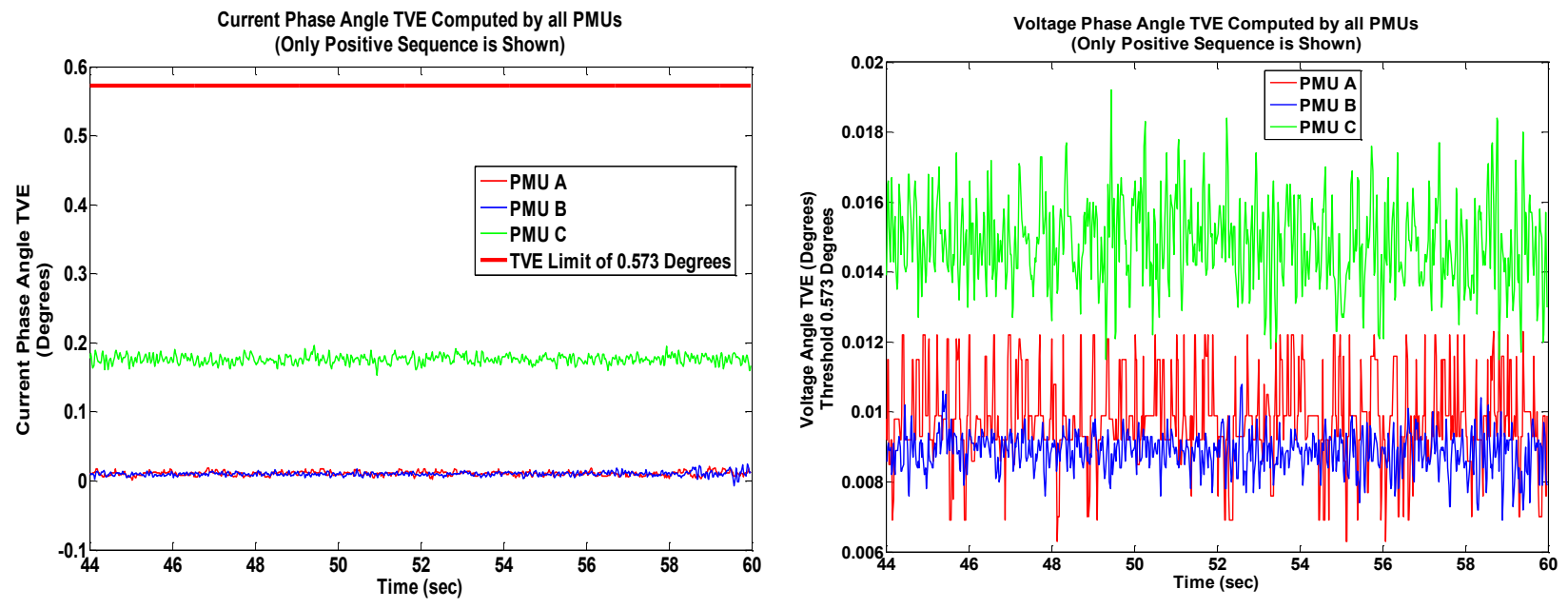

Fig. 4. Voltage and Current Phase Angle TVE computed by all the PMUs under test when subjected to balanced three phase $60 \mathrm{~V}$ and 1 Amp injections. Only angle TVE for positive sequence voltage and current is shown.

\section{B. Steady-State with Harmonic Distortion}

In order to further evaluate the performance of PMUs under steady state, harmonic distortion was added to the three phase voltage and current injections to the PMUs.

The IEEE C37.118.1 standard [3] specifies that the maximum TVE for magnitude and phase angle calculations for both voltage and current measurements by a PMU should be lesser than $1 \%$ even if the input signal contains up to the $50^{\text {th }}$ harmonic of the amplitude of $1 \%$ of the nominal input voltage and current. The Freja-300 test set facilitates to inject harmonic distortions in both voltage and current signals for up to the $25^{\text {th }}$ harmonic. By using Freja-Win [6], the voltage and current injections can be defined by using a mathematical expression of the form;

$$
\text { Amplitude }=100 \% \times \sin (2 \times \pi * f)+1 \% \times \sin (2 \times \pi * f * n)
$$

where ' $f$ ' is the frequency of the fundamental component which in this study is $50 \mathrm{~Hz}$ and ' $n$ ' is the order of harmonic. As the input voltage and current signals being generated by the test set are 60 Volts and 1 Ampere respectively, (3) yields the following mathematical expression for both voltage and current injections with harmonic distortions

$$
\begin{aligned}
& \text { Amplitude }_{\text {Voltage }}=60 \times \sin (2 \times \pi * 50)+0.6 \times \sin (2 \times \pi * 50 * n) \\
& \text { Amplitude }_{\text {Current }}=1 \times \sin (2 \times \pi * 50)+0.01 \times \sin (2 \times \pi * 50 * n)
\end{aligned}
$$

All the tests performed with balanced three phase signals were performed again with the distorted signals. The performance of PMUs was evaluated with the same criteria for TVE, FE and RFE tolerance. Fig. 5 shows the plots of the TVE computed for both positive sequence and phase measurements of voltage (plots a-c) and current magnitudes (plots d-f) for the three PMUs when subjected to distorted voltage and current inputs. The results show that all the PMUs have TVE lesser than $1 \%$ for both voltage and current magnitudes for all the phases. However they are larger than the balanced three phase scenario. If the order of harmonic content is increased, the TVE increases more significantly for current magnitudes as compared to voltage magnitudes. Fig. 6 (a) shows the current magnitude TVE for PMU ' $\mathrm{C}$ ' when $1 \%$ of the $25^{\text {th }}$ order har- 
monic is injected in the input test signal. Similarly if the amplitude of the harmonics is increased, TVE for current magnitude increases significantly as compared to voltage magnitude TVE. Fig. 6 (b) shows the current magnitude of TVE calculated for PMU ' $\mathrm{C}$ ' when input signal has $7 \% 3^{\text {rd }}$ harmonic content. By comparing it with Fig. 5(f), the significant increase in TVE is obvious. However the effect of harmonic order and harmonic amplitude on FE is marginal.

Fig. 7 shows the results for both frequency measurement error (FE) and rate of change of frequency (ROCOF) measurement error (RFE) under harmonic distortion. All the PMUs satisfy the criteria of steady state frequency error (FE) (within $\pm 0.005 \mathrm{~Hz}$ ). PMU 'A' does show an $\mathrm{FE}$ outside $\pm 0.005 \mathrm{~Hz}$ limit but that is because of the missing data reported by the PDC which fills the missing data with zeros. In case of RFE, both PMU 'B' and PMU ' $\mathrm{C}$ ' violates the maximum allowable limit of $\pm 0.01 \mathrm{~Hz} / \mathrm{sec}$. This violation is due to the presence of harmonics in the input voltage and current magnitudes and phase angles. PMU ' $A$ ' fulfils the requirement for almost the entire test run except for few instances of missing data.

Fig. 8 shows the results for Voltage and Current phase angle TVE for system with harmonic distortions. TVE for all the PMUs is lesser than the maximum permissible tolerance of $0.573^{\circ}$. However, TVE for both voltage and current phase angles have increased due to the presence of harmonic distortion.
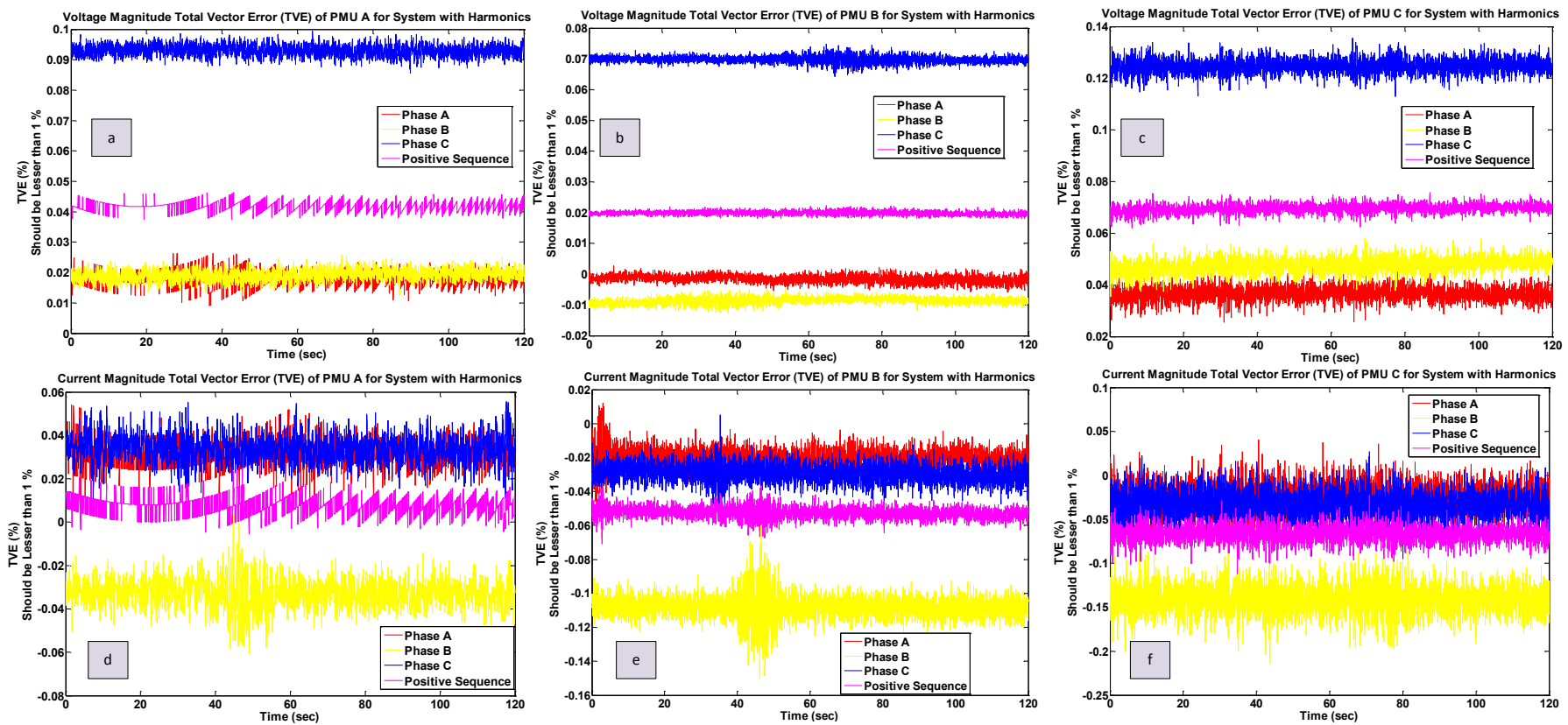

Fig. 5. Voltage and current TVE computed from the measurements of three PMUs under test in steady state conditions when subjected to harmonic distortions. (1). Plot (a-c) show voltage magnitude TVE for all phases including positive sequence for the three PMUs when signal contains a $3^{\text {rd }}$ harmonic equal to $1 \%$ of nominal voltage. Similarly plots (d-f) show current magnitude TVE for distorted input signals.
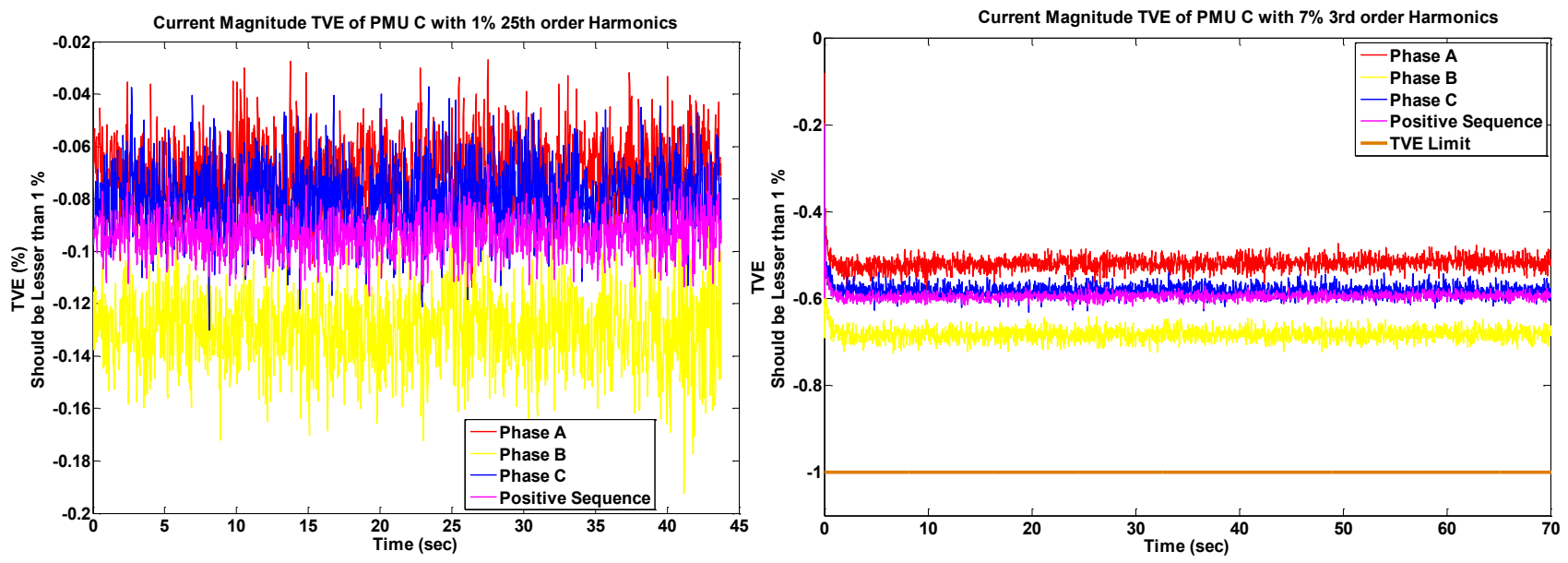

Fig. 6. Current magnitude TVE of PMU C when (a) the input signal has $1 \% 25^{\text {th }}$ order harmonics and (b) when input signal has $7 \% 3^{\text {rd }}$ order harmonics. Compare it with plot in Fig. 5(f) to note the major differences in TVE due to harmonics. 

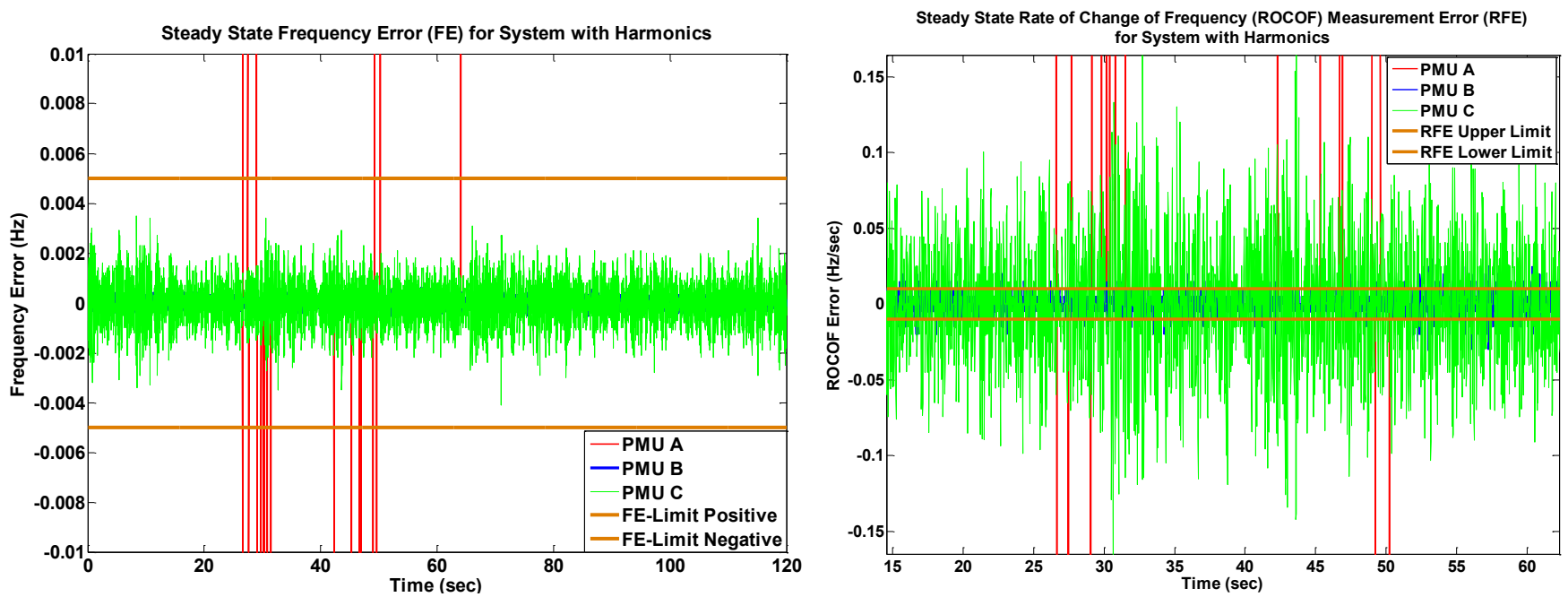

Fig. 7. Frequency Error (FE) and Rate of Change of Frequency (ROCOF) measurement error (RFE) for steady state system with $3^{\text {rd }}$ harmonics of $1 \%$ of the nominal injected voltage and current. All the PMUs satisfy the requirement for frequency error within $\pm 0.005 \mathrm{~Hz}$. Only PMU 'A' satisfies the requirement for RFE. PMU 'A' has reports missing data due to which it violates the limit occasionally.
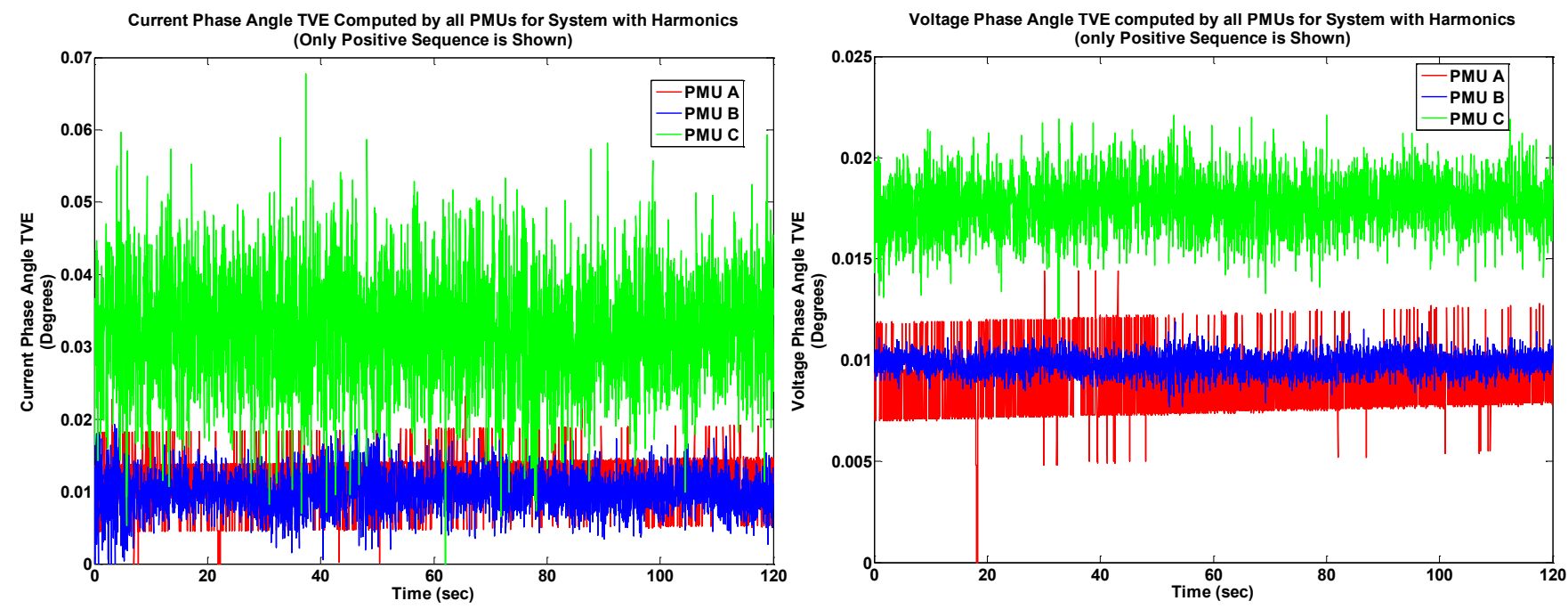

Fig. 8. Voltage and Current Phase Angle TVE computed by all the PMUs under test when subjected to voltage and current inputs with $3^{\text {rd }}$ harmonic. Only phase angle TVE for positive sequence voltage and current is shown for all PMUs

On the basis of TVE, FE and RFE computed for both balanced system and system with harmonic distortions, the PMUs are assigned either pass or fail grade. These results are summarized in Table 1. All the three PMUs fulfil most of the performance criteria under steady state conditions with no harmonic distortions; however with harmonic distortions two PMUs violate the RFE limit.

TABLE I

PMU STEAdy State Performance Analysis

\begin{tabular}{|c|c|c|c|c|c|c|c|c|}
\hline Test & \multicolumn{3}{|c|}{ Balanced Three Phase } & \multicolumn{5}{c|}{ Harmonics Injection } \\
\hline PMUs & $\begin{array}{c}\text { TVE } \\
(\text { Mag })\end{array}$ & $\begin{array}{c}\text { TVE } \\
(\text { Phase })\end{array}$ & FE & RFE & $\begin{array}{c}\text { TVE } \\
(\text { Mag) }\end{array}$ & $\begin{array}{c}\text { TVE } \\
(\text { Phase })\end{array}$ & FE & RFE \\
\hline PMU A & $\mathrm{P}$ & $\mathrm{P}$ & $\mathrm{P}$ & $\mathrm{P}$ & $\mathrm{P}$ & $\mathrm{P}$ & $\mathrm{P}$ & $\mathrm{P}$ \\
\hline PMU B & $\mathrm{P}$ & $\mathrm{P}$ & $\mathrm{P}$ & $\mathrm{P}$ & $\mathrm{P}$ & $\mathrm{P}$ & $\mathrm{P}$ & $\mathrm{F}$ \\
\hline PMU C & $\mathrm{P}$ & $\mathrm{P}$ & $\mathrm{P}$ & $\mathrm{F}$ & $\mathrm{P}$ & $\mathrm{P}$ & $\mathrm{P}$ & $\mathrm{F}$ \\
\hline
\end{tabular}

\section{Limitations of PMU Testing EQUiPMENT}

Almost all the steady state tests recommended in the IEEE C37.118.1 [3] can be performed with stand-alone test set. However, some of the limitations involve the injection of relevant harmonic distortions. The standard emphasizes that any harmonic (up to 50th) at $1 \%$ of signal magnitude should not result in TVE exceeding 1\%. However, the Freja-300 test set is only capable of injecting up to $25^{\text {th }}$ harmonic. Another limitation is when performing off-nominal frequency range steady state tests because the accuracy of test set for offnominal frequency is also arguable. Based on these limitations, it is recommended to perform real-time simulation with the PMU as hardware-in-the-loop (HIL) for comprehensive testing of PMUs. KTH SmarTS-Lab [9] is equipped with Opal-RT's eMEGAsim Real-Time Simulator and RT-HIL testing can be performed for the calibration testing of PMUs 
according to the standard. The model-to-data workflow for performing real-time hardware-in-the-loop (RT-HIL) compliance testing of PMUs with Opal-RT's eMEGAsim Real-Time Simulator (RTS) is shown in Figure 9. The three phase voltage and current signals are modelled using SimPowerSystem's library of MATLAB/Simulink. The model is executed in realtime using Opal-RT's real-time targets. The low-level analog signals of voltages and currents are accessed from the analog outputs of RTS and are amplified using linear amplifiers. The amplified three phase voltage and current signals are fed to the VT and CT input of the PMU under test respectively.
The accuracy of the PMU measurements can be evaluated by comparing the reference signals (generated by the Simulink model) with the event reports retrieved from the PMU which contains the detail of different analog/digital/phasor signals as measured by the PMU. In this way a more thorough compliance testing of a PMU can be performed as the Simulink based mathematical model can generate any number of harmonics. In this approach, it is important to characterize the error in the $\mathrm{D} / \mathrm{A}$ interface between the simulator and the amplifiers, which if not done properly, may affect directly the compliance test results. The authors will present results on this approach, and discuss challenges and limitations in a separate publication.

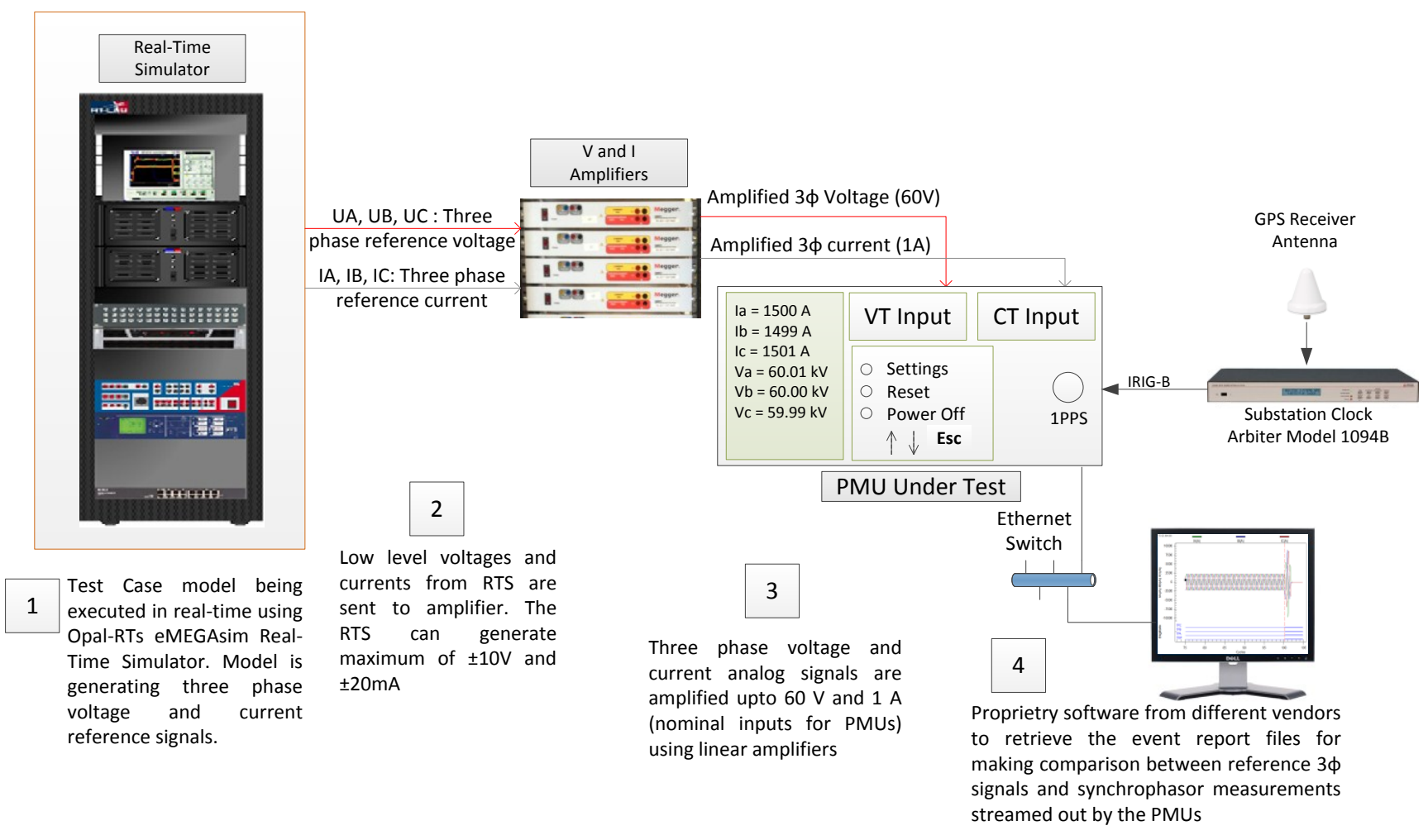

Fig. 9. Steps involved in Real-Time Hardware-in-the-Loop (RT-HIL) steady-state / dynamic compliance testing of PMU

Freja-300 test set does not have the provision for time synchronization and transmitting this timing signal to the device under test. Methodologies are currently being investigated to synchronize Freja-300 and PMU with the same timing source in order to have the whole PMU testing procedure as time synchronized.

With the same test set, dynamic compliance testing of the same PMUs is also performed. One of the results from the dynamic compliance testing is shown in Fig. 10, where a $10 \%$ step is applied to the input current magnitude of the three PMUs. The plot shows that PMU A has a slow response while PMU C has a large overshoot. All the dynamic tests performed will be reported as a separate publication.

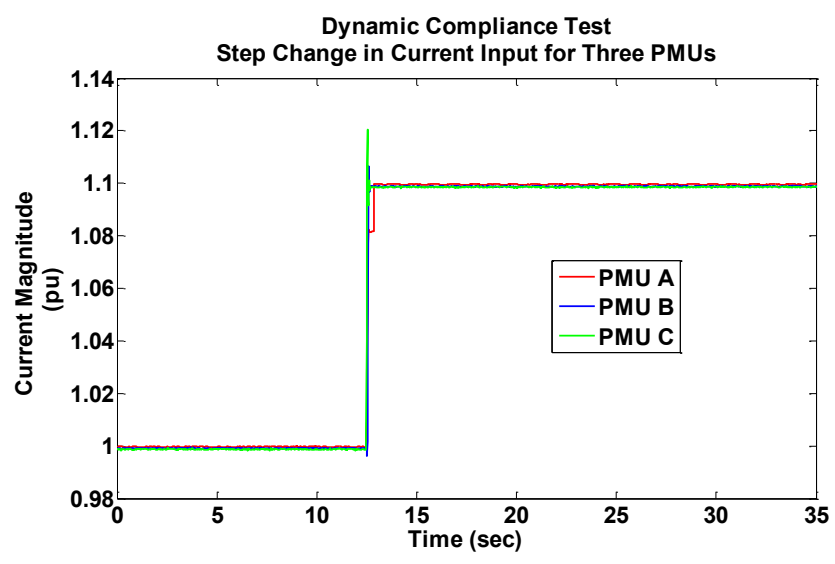

Fig. 10. PMUs response for dynamic compliance testing when subjected to $10 \%$ current magnitude step 


\section{CONCLUSION}

The PMU static compliance testing process is presented for testing the accuracy of PMUs from three different vendors according to the requirements specified in the standard IEEE C37.118.1-2011 and IEEE C37.242-2013. Various aspects of calibration and testing of these PMUs with the Freja-300 test set have been discussed in this paper along with the limitations of using stand-alone testing equipment. The comparison of these PMUs is made on the basis of the Total Vector Error (TVE), Frequency Error (FE), Rate of change of Frequency (ROCOF) measurement Error (RFE) calculations. The PMU performance is evaluated for both balanced three phase system and system with harmonic distortions.

TVE, FE and RFE for balanced three phase signals are well within the maximum allowable tolerance specified by the standard. However, when harmonic content is added in the test signals, TVE, FE and RFE value increases - still, they remain within the limit for nominal frequency. TVE for voltage magnitude is much smaller than for current magnitude. However, TVE for current magnitude increases significantly as compared to voltage magnitude TVE for higher order and higher amplitude of harmonic contents. Two of the PMUs in the study violated the RFE limits under harmonic distortion test in steady state.

\section{REFERENCES}

[1] A. Bose, "Smart Transmission Grid Applications and Their Supporting Infrastructure," IEEE Trans on Smart Grid, vol. 1, pp. 11 -19, 2010.

[2] D. E. Bakken, A. Bose, C. H. Hauser, D. E. Whitehead, and G. C. Zweigle, "Smart Generation and Transmission with Coherent, Real-Time Data," Proceedings of the IEEE, vol. 99, no. 6, pp. 928 - 951, 2011.

[3] IEEE C37.118-2005, IEEE Standard for Synchrophasors in Power Systems.

[4] IEEE Standard C37.242-2013 - IEEE Guide for Synchronization, Calibration, Testing, and Installation of Phasor Measurement Units (PMUs) for Power System Protection and Control, IEEE Standards Association, March 2013

[5] Megger, "Freja 300 - Relay Test System," available on-line: http://tinyurl.com/Freja300.

[6] Megger, "Freja Win- Graphical interface for Freja-300 test set", available on-line: http://tiny.cc/Freja-Win

[7] IRIG Standard 200-04, IRIG serial time code format, September 2004, available online: http://www.irigb.com/pdf/wp-irig-200-04.pdf

[8] Arbiter, "GPS Substation Clock - Model 1094B", available online:http://www.arbiter.com/catalog/product/model-1094b-gps-satelliteprecision-time-clock.php

[9] M. S. Almas et al., "Synchrophasor Network, Laboratory and Software Applications Developed in the STRONg ${ }^{2}$ rid Project", IEEE Power and Energy Society, 2014 General Meeting, July 2014.

\section{BIOGRAPHIES}

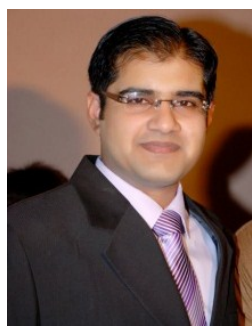

Muhammad Shoaib Almas (Student Member '12) has received the B.Sc. degree in Electrical Engineering from National University of Sciences and Technology (NUST), Pakistan, in 2007, and the M.Sc. degree in Electric Power Engineering from $K T H$ Royal Institute of Technology, Stockholm, Sweden, in 2011. He is currently a Ph.D. student within the Electric Power Systems (EPS) Division at KTH. The main theme of his current research is the "PMUassisted real-time distributed control of hybrid $\mathrm{AC}$ and DC grids for damping inter-area electromechanical oscillations". He has a two year professional experience in substation automation and power system protection desgining using microprocessor based protection relays (GE Multilin/GE Energy). His research interests are wide area power system monitoring, protection, automation and control, communication network simulation and cyber security.

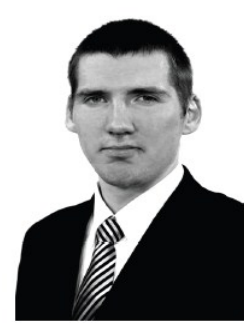

Jako Kilter (Student Member '08, M'10) was born in Tallinn, Estonia, 1981. He received the B.Sc., M.Sc. and $\mathrm{PhD}$ degrees in electrical engineering from Tallinn University of Technology (TUT), Tallinn, Estonia, in 2003, 2005, and 2009, respectively. Currently he is working at the Department of Electrical Power Engineering of TUT as an associate professor and at the Estonian Transmission System Operator as a power system expert. He has published more than 25 papers on load modelling, system control and power quality and is the author of three textbooks. He is a Chartered Engineer, Chairman of Estonian Centre for Standardization Committee of High Voltage Engineering, and member of different ENTSO-E and CIGRE working groups.

His special field of interest include electrical network state estimation and analysis, power system monitoring and control, modelling of loads, power system stability, power quality, HVDC and FACTS.

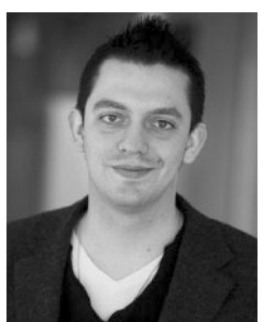

Luigi Vanfretti (Student Member '03, M'10, SM'14) received the electrical engineering degree from Universidad de San Carlos de Guatemala, Guatemala City, Guatemala, in 2005, and the M.Sc. and $\mathrm{Ph} . \mathrm{D}$. degrees in electric power engineering from Rensselaer Polytechnic Institute, Troy, NY, USA, in 2007 and 2009, respectively.

He was a Visiting Researcher with The Univer- sity of Glasgow, Glasgow, Scotland, in 2005. He became an Assistant Professor with the Electric Power Systems Department, KTH Royal Institute of Tech- nology, Stockholm, Sweden, in 2010 and was conferred the Swedish title of "Docent" in 2012. He is currently a tenured Associate Professor with the same department.

$\mathrm{He}$ is Special Advisor in Strategy and Public Affairs for the Research and Development Division of Statnett SF, the Norwegian transmission system operator. His duties include architectural analysis for synchrophasor data transfer, communications, and application sys- tems to be utilized in Smart Transmission Grid applications; as well as providing inputs into R\&D strategy development and aiding in the execution of collabo- rative projects with universities, TSOs, and R\&D providers. He is an advocate for free/libre and open-source software. His research interests are in the general area of power system dynamics; while his main focus is on the development of applications of PMU data.

Dr. Vanfretti has served, since 2009, in the IEEE Power Engineering Society (PES) PSDP Working Group on Power System Dynamic Measurements, where he is now Vice-Chair. In addition, since 2009, he has served as Vice-Chair of the IEEE PES CAMS Task Force on Open Source Software. He is an evangelist of Free/Libre and Open Source Software for power system engineering education; he is Associate Member of the Free Software Foundation and member of the Open Source Modelica Consortium.

For his research and teaching work toward his Ph.D. degree, he was awarded the Charles M. Close Award from Rensselaer Polytechnic Institute. 